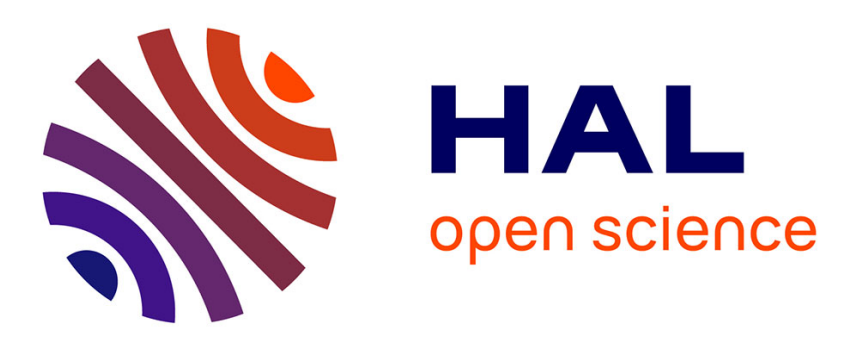

\title{
Emerging Mechanisms by which EMT Programs Control Stemness
}

Molly M Wilson, Robert A Weinberg, Jacqueline A Lees, Vincent J Guen

\section{To cite this version:}

Molly M Wilson, Robert A Weinberg, Jacqueline A Lees, Vincent J Guen. Emerging Mechanisms by which EMT Programs Control Stemness. Trends in Cancer, 2020, 6 (9), pp.775-780. 10.1016/j.trecan.2020.03.011 . hal-02565570

\section{HAL Id: hal-02565570 \\ https://hal-univ-rennes1.archives-ouvertes.fr/hal-02565570}

Submitted on 20 May 2020

HAL is a multi-disciplinary open access archive for the deposit and dissemination of scientific research documents, whether they are published or not. The documents may come from teaching and research institutions in France or abroad, or from public or private research centers.
L'archive ouverte pluridisciplinaire HAL, est destinée au dépôt et à la diffusion de documents scientifiques de niveau recherche, publiés ou non, émanant des établissements d'enseignement et de recherche français ou étrangers, des laboratoires publics ou privés. 


\section{Emerging mechanisms by which EMT programs control stemness}

Molly M. Wilson1, Robert A. Weinberg1,2, Jacqueline A. Lees1, Vincent J. Guen3

${ }_{1}$ Koch Institute for Integrative Cancer Research and Department of Biology, Massachusetts Institute of Technology, Cambridge, MA, USA 2Whitehead Institute, Cambridge, MA, USA 3Univ Rennes, CNRS, IGDR (Institut de génétique et développement de Rennes)UMR 6290, F- 35000 Rennes, France.

Correspondence: vincent.guen@univ-rennes1.fr (V.J. Guen) Twitter: Vincent GUEN @VincentGuen

Tissue regeneration relies on adult stem cells (SCs) that possess the ability to self-renew and produce differentiating progeny. In an analogous manner, the development of certain cancers depends on a subset of tumor cells, called cancer stem cells (CSCs), with SC-like properties. In addition to being responsible for tumorigenesis, CSCs exhibit elevated resistance to therapy and thus drive tumor relapse post-treatment. The epithelial-mesenchymal transition (EMT) programs promote SC and CSC stemness in many epithelial tissues. Here, we provide an overview of the mechanisms underlying the relationship between stemness and EMT programs, which may represent therapeutic vulnerabilities for the treatment of cancers.

Keywords: EMT; cancer stem cells; stemness.

\section{Epithelial-Mesenchymal Transition}

Epithelial-mesenchymal transition (EMT, see Glossary) is a cell-biological program enabling an epithelial cell to acquire an array of mesenchymal phenotypes [1]. During this process, an epithelial cell that displays a cobblestone morphology progressively adopts an elongated appearance. Compelling evidence of interconversion between the epithelial and mesenchymal states came in the 1980s from pioneering observations in the developing chick embryo [2-4]. Initially called the epithelial-mesenchymal 
transformation, it was soon renamed as a transition in order to distinguish it from neoplastic transformation and to emphasize the reversible nature of this program [1]. Successive rounds of EMT and the reverse process, termed mesenchymal-epithelial transition (MET), occur during embryonic development [1].

EMT was initially described as a process inducing motile properties in embryonic cells that enable, in turn, specific steps of morphogenesis [1]. The study of developmental events, including gastrulation and neural crest cell delamination, led to the identification of core EMT-inducing transcription factors (EMT-TFs), including Snail, Twist, and Zeb, each of which comes in at least two variants [5]. The specific roles of individual EMT-TFs, which are responsible for orchestrating various EMT programs, vary across tissues and species [5].

Beyond their key developmental role, EMT programs were also shown in early studies to confer motile properties on cells within adult tissues of various organisms, doing so in both physiological and pathological settings [6]. For example, EMT programs promote wound healing in the skin [7], ovary [8], and during heart regeneration postinjury [6, 9]. EMT programs are also activated in various disease states. During tissue degeneration associated with organ fibrosis, EMT programs enable epithelial cells to induce the formation of myofibroblasts in nearby stroma [6]. These cells accumulate within this compartment, where they secrete pro-fibrotic factors that sustain disease progression and ultimately contribute to organ failure [6].

Soon after the discovery of EMT, some hypothesized that activation of this cellbiological program might be exploited by carcinoma cells to enable their motility and lead, in turn to metastatic dissemination [2]. Evidence supporting this idea first came from in vitro work showing that cells of carcinoma cell lines with invasive properties exhibit a number of mesenchymal phenotypes and, at the same time, express low levels of the canonical epithelial marker and cell junction component, E-cadherin [10]. Subsequently, germline and somatic loss-of-function mutations in the E-cadherin gene $(C D H 1)$ were identified in cells of human invasive carcinomas [11-13].

These observations provided compelling support for the notion that the disruption of cell junction integrity enabled increased invasive capacity of carcinoma cells. However, 
almost a decade passed before EMT and, more specifically, expression of a canonical EMT-TF, Twist, was proven to promote metastasis in a murine mammary carcinoma model [14]. In the time since this observation, the link between EMTs, induced by various EMT-TFs, and metastasis has been established in many different tumor types both in vitro and in vivo [15-17]. During the course of these studies, it became apparent that EMT programs do not operate as simple binary switches that toggle between epithelial and mesenchymal phenotypic states. Instead, we now know that EMT can generate a spectrum of cellular states displaying mixed epithelial and mesenchymal features that lie between these two extremes in vitro and in vivo. Notably, only a subset of these phenotypically mixed states is associated with metastatic dissemination [1, 18] (Figure 1, Key Figure). The critical role of EMT in inducing cell motility and metastasis, as well as its underlying molecular mechanisms, have been extensively reviewed elsewhere [15]. The goal of the present article is to discuss another set of actions coordinated by EMT programs that has more recently emerged. Here, we review the underlying mechanisms that support the notion that EMT programs induce stemness in normal and neoplastic cells of normal tissues and carcinomas.

\section{EMT and Activation of Stemness}

Although the original focus on the roles of EMT shed light on its pro-invasive properties, work over the past decade revealed that it also induces SC properties [19]. This was first demonstrated in the context of the mammary gland. Populations enriched for either mouse or human non-neoplastic mammary SCs (MaSCs) were shown to express EMT markers, and induction of EMT in mammary epithelial cells caused expression of SC markers and enabled in vitro mammosphere- or tumorsphere-forming capabilities of normal and neoplastic mammary epithelial cells, respectively [20, 21]. Moreover, EMT induction was shown to increase the tumor-initiating capacity of transformed cells in a murine mammary tumor model [20].

Further analyses of the normal mammary gland revealed that the Slug EMT-TF (also known as Snail2) is a key inducer of MaSC stemness [22, 23]. Slug is expressed within the basal cell population in the mammary epithelium that is enriched for MaSCs $[20,22-25]$, and the self-renewal capacity of these cells in organoid and transplantation/reconstitution assays is enhanced or suppressed by Slug 
overexpression or knockdown, respectively [22, 25]. Indeed, Slug inhibition promotes luminal epithelial differentiation $[24,26]$ and, accordingly, Slug-knockout mice show a delay in mammary gland development that reflects a defect in lineage commitment [23, 24]. These Slug-knockout mice are resistant to MMTV-Myc-induced tumorigenesis, reinforcing the requirement for EMT in tumorigenesis [24]. Yet other studies have shown that other EMT-TF inducers, including Twist, Six1 and Snail, promote CSC stemness and tumorigenesis in various autochthonous mouse models of breast cancer development $[25,27,28]$. To summarize, the association of EMT programs with stemness, at least in the context of mammary cells, applies to both normal and neoplastic cells.

\section{Mechanisms of EMT-Induced -Stemness}

A number of studies have now established that EMT programs induce stemness in many tissues and cancer types including the mammary gland, pancreas, colon, head and neck cancer, lung, prostate, and ovary [19]. Although there is extensive information about the mechanisms that induce either EMT or stemness, remarkably few studies address precisely how, mechanistically, EMT programs induce stemness. Here, we review specific candidate signaling pathways that have been elucidated in the context of studies of the breast, pancreas and colon [29-34]. While there is not one universally applicable mechanism, all culminate in activation of signaling pathways and/or epigenetic regulators that are well-established SC regulators.

The first mechanistic link between EMT and stemness comes from analysis of the EMT-TF Zeb1 in pancreatic cancer [29]. The authors began by confirming that Zeb1 was required for stemness by showing that its knockdown causes pancreatic cell lines to lose their tumorsphere-forming capacity in vitro and their tumor-initiating ability in vivo. They then established that Zeb1 directly represses several miRNAs of the miR200 family, which were earlier found to repress CSC stemness in other settings [35], by binding to their upstream promoters. Overexpression or inhibition of these miRNAs resulted in decreased or increased tumorsphere-forming capacity, respectively, confirming that they act to repress stemness of mouse and human pancreatic CSCs. Importantly, these miRNAs were shown to directly repress the expression of the epigenetic regulator Bmi1, which is known to play a key role in maintaining adult SC function [36, 37]. Hence, these results revealed a direct Zeb1/miR-200/Bmi1 pathway 
in pancreatic CSCs that explains, at least in part, how EMT enables stemness in this setting.

In the context of head and neck squamous cell carcinoma (HNSCC), an independent study discovered that a second EMT-TF, Twist, signals to Bmi1 to control CSC stemness [30]. In this case, the investigators overexpressed Twist, Snail and Slug (=Snail2) in HNSCC cell lines and examined the impact of each on transcription of a set of known SC regulators; they found that Twist activates Bmi1 and directly binds its promoter. Twist and Bmi1 were both shown to induce tumorsphere and tumor-initiating properties of HNSCC cells, confirming that they play key roles in stemness. Moreover, Bmi1 is critical to Twist's stemness-inducing ability in these cells, since its knockdown was found to ablate Twist-induced tumorsphere- and tumor-initiating capacity.

Interestingly, further characterization suggested that Bmi1 acts in concert with Twist, and not simply downstream of Twist, in these HNSCC cell lines. First, Bmi1 was found, on its own, to be capable of inducing an EMT. Second, Twist and Bmi1 were found to physically interact with one another, and to bind to the same promoter regions of canonical Twist (Cdh1) and Bmi1 (Cdkn2a) target genes, repressing their expression. Given these findings, one might predict that Twist is necessary for Bmi1 to promote stemness in the HNSCC cells, but this has yet to be tested directly.

Independent work highlighted how signaling between EMT regulators and miR-200 family members promotes CSC stemness. The first study elucidated a Zeb1/miR200/Notch signaling axis [32]. Zeb1 was found to inhibit miR-200 family members, resulting in de-repression of expression of the Notch signaling components Jag1, Mam/2 and Mam/3, which are direct targets of miR-200 inhibition in breast and pancreatic cancer cell lines. Activation of Notch signaling was shown to induce stemness of CSCs, since Notch pathway inhibition - achieved either by treatment with a $\gamma$-secretase inhibitor or by Jag1/Maml2/Maml3 knockdown - reduced the tumorsphere-forming capacity of pancreatic cancer cells. Interestingly, Jag1 knockdown also decreased Zeb1 expression, suggesting that Jag1 and Zeb1 are involved in a reciprocal positive-feedback loop in which EMT and stemness reinforce expression of one another. 
A second study elucidated other downstream events of the EMT/miR-200 axis in normal mammary SCs, which promotes asymmetric division and thus maintenance of the mammary SC pool [34]. Specifically, the repression of miR-200c by EMT programs caused de-repression of one of its direct targets, PGC1 $\alpha$, which acts as a transcriptional co-activator of expression of the gene encoding MFN1. MFN1, for its part, is a component of a tri-partite complex, MFN1-PKC $\zeta-N U M B$, that controls asymmetric cell division. PKC $\zeta$ knockdown was found to inhibit the TGF $\beta$-induced asymmetric division of mammary SCs, and MFN1 knockdown suppressed the TGF $\beta$ induced mammosphere-forming capacity of these SCs. Asymmetric cell division, a critical component of SC biology, was associated with asymmetric allocation of fused mitochondria to the daughter cell that maintains SC properties (rather than the daughter that proceeds to generate more differentiated progeny). The stem cell daughter thereby maintains metabolic properties that enable the maintenance of its self-renewal capacity. Consistent with these in vitro results, mir-200c knockout in the mouse mammary gland induced expansion of mammary SCs exhibiting mammosphere- and mammary gland-reconstituting abilities.

Another study supported the role of yet other miRNAs in EMT-induced acquisition of stemness [31]. Thus, in the colorectal cancer cell lines under study, Snail was observed to be the most highly expressed EMT-TF, and shown to induce both tumorsphere- and tumor-initiating capacity. Molecular analyses revealed that Snail binds and activates the promoter of miR-146a, which represses the expression of NUMB, an inhibitor of the Wnt signaling effector protein, $\beta$-catenin. This sequence of molecular events promotes activation of the Wnt pathway, which is a well-documented regulator of stemness. Importantly, the authors showed that miR-146 and NUMB both influence tumorsphere formation, validating the role of this Snail/miR-146/NUMB/ $\beta$ catenin pathway in promoting SC properties. Moreover, they established its clinical relevance by showing that a SnailHigh NUMBLow profile correlates with poor prognosis and therapy resistance in a cohort of colorectal cancer patients.

It is interesting to note that studies in a different context-breast cancer-have reported a positive feedback loop between Wnt signaling and Bmi1, raising the possibility that Bmi1 is also a component of this Wnt pathway-induced stemness. 
A more recent study established that EMT programs can induce stemness in the mammary gland and in breast cancers by inducing primary ciliogenesis [33]. Primary ciliogenesis is the dynamic process of assembling the primary cilium, which is a single microtubule-based protruding structure that is assembled at the surface of a cell. Frequently called the cell's antenna, the primary cilium has been recognized to act as a cell signaling center in cells found in a variety of tissues [38]. In this work, it was initially shown that primary cilia are observed on Slug-expressing cells in the SCenriched compartment of the normal mammary gland, as well as in mesenchymal-like, EMT-TF-expressing cells within a murine carcinoma model of human Claudin-low breast cancer. This study also demonstrated that activation of an EMT program, achieved either through forced expression of various EMT-TFs (Snail, Twist or Zeb1) or knockdown of E-cadherin, induces primary ciliogenesis in both normal and transformed mammary epithelial cells. The resulting primary cilia were shown to coordinate Hedgehog signaling, a pathway that has been independently linked to the induction of stemness of both normal and neoplastic mammary epithelial cells, whose activation was previously associated with EMT program activation [39, 40]. Importantly, genetic inhibition of primary ciliogenesis resulted in the loss of organoid-forming capacity of normal mouse mammary SCs and of tumor-initiating capacity of breast cancer cells as gauged by orthotopic implantation assays. Altogether, these data established an ordered pathway of EMT/primary ciliogenesis/Hedgehog signaling/stemness, and showed that primary cilia are required for stemness in these contexts. Finally, we note that Hedgehog has been linked to the regulation of Bmi1 [39]. This raises the intriguing possibility that Bmi1 plays a frequent and widespread role in the induction of stemness by EMT, doing so in a variety of biological contexts.

\section{Concluding Remarks}

EMT program activation has been linked over the past decade to induction of SC properties in both normal and transformed cells residing in many tissues [19]. To date, several studies have directly addressed how EMT itself induces stemness, and specifically in the mammary gland, pancreas and colon [29-34]. These studies reveal various signaling pathways that all lead to the activation of well-established SC regulators. The roles of these pathways in the induction of SC and CSC stemness in other tissues remain to be explored, and the contribution of additional intracellular 
signaling pathways awaits further investigation. Since CSCs appear to be responsible for tumor initiation as well as for post-treatment relapses in the oncology clinic, a better characterization of these mechanisms may reveal new molecular vulnerabilities in CSCs (see outstanding questions) that could be targeted to improve cancer therapeutics.

\section{Acknowledgements}

The work of the authors has been supported by grants from Fondation MIT, Fondation ARC, Cancéropôle Grand Ouest, Université de Rennes 1.

\section{References}

1 Nieto, M.A., et al. (2016) Emt: 2016. Cell 166, 21-45

2 Thiery, J.P. (2002) Epithelial-mesenchymal transitions in tumour progression. Nat Rev Cancer 2, 442-454

3 Trelstad, R.L., et al. (1967) Cell contact during early morphogenesis in the chick embryo. Dev Biol 16, 78-106

4 Greenburg, G. and Hay, E.D. (1982) Epithelia suspended in collagen gels can lose polarity and express characteristics of migrating mesenchymal cells. J Cell Biol 95, 333-339

5 Stemmler, M.P., et al. (2019) Non-redundant functions of EMT transcription factors. Nat Cell Biol 21, 102-112

6 Thiery, J.P., et al. (2009) Epithelial-mesenchymal transitions in development and disease. Cell 139, 871-890

7 Arnoux, V., et al. (2008) Erk5 controls Slug expression and keratinocyte activation during wound healing. Mol Biol Cell 19, 4738-4749

8 Ahmed, N., et al. (2006) Molecular pathways regulating EGF-induced epitheliomesenchymal transition in human ovarian surface epithelium. Am J Physiol Cell Physiol 290, C1532-1542

9 Lepilina, A., et al. (2006) A dynamic epicardial injury response supports progenitor cell activity during zebrafish heart regeneration. Cell 127, 607-619

10 Frixen, U.H., et al. (1991) E-cadherin-mediated cell-cell adhesion prevents invasiveness of human carcinoma cells. J Cell Biol 113, 173-185

11 Becker, K.F., et al. (1994) E-cadherin gene mutations provide clues to diffuse type gastric carcinomas. Cancer Res 54, 3845-3852

12 Berx, G., et al. (1995) E-cadherin is a tumour/invasion suppressor gene mutated in human lobular breast cancers. EMBO J 14, 6107-6115

13 Guilford, P., et al. (1998) E-cadherin germline mutations in familial gastric cancer. Nature 392, 402-405

14 Yang, J., et al. (2004) Twist, a master regulator of morphogenesis, plays an essential role in tumor metastasis. Cell 117, 927-939

15 Lambert, A.W., et al. (2017) Emerging Biological Principles of Metastasis. Cell 168, 670-691 
16 Pastushenko, l., et al. (2018) Identification of the tumour transition states occurring during EMT. Nature 556, 463-468

17 Pattabiraman, D.R. and Weinberg, R.A. (2014) Tackling the cancer stem cells what challenges do they pose? Nat Rev Drug Discov 13, 497-512

18 Pastushenko, I. and Blanpain, C. (2019) EMT Transition States during Tumor Progression and Metastasis. Trends Cell Biol 29, 212-226

19 Dongre, A. and Weinberg, R.A. (2019) New insights into the mechanisms of epithelial-mesenchymal transition and implications for cancer. Nat Rev Mol Cell Biol 20, 69-84

20 Mani, S.A., et al. (2008) The epithelial-mesenchymal transition generates cells with properties of stem cells. Cell 133, 704-715

21 Morel, A.P., et al. (2008) Generation of breast cancer stem cells through epithelialmesenchymal transition. PLoS One 3, e2888

22 Guo, W., et al. (2012) Slug and Sox9 cooperatively determine the mammary stem cell state. Cell 148, 1015-1028

23 Nassour, M., et al. (2012) Slug controls stem/progenitor cell growth dynamics during mammary gland morphogenesis. PLoS One 7, e53498

24 Phillips, S., et al. (2014) Cell-state transitions regulated by SLUG are critical for tissue regeneration and tumor initiation. Stem Cell Reports 2, 633-647

$25 \mathrm{Ye}, \mathrm{X}$., et al. (2015) Distinct EMT programs control normal mammary stem cells and tumour-initiating cells. Nature 525, 256-260

26 Proia, T.A., et al. (2011) Genetic predisposition directs breast cancer phenotype by dictating progenitor cell fate. Cell Stem Cell 8, 149-163

27 McCoy, E.L., et al. (2009) Six1 expands the mouse mammary epithelial stem/progenitor cell pool and induces mammary tumors that undergo epithelialmesenchymal transition. J Clin Invest 119, 2663-2677

28 Morel, A.P., et al. (2012) EMT inducers catalyze malignant transformation of mammary epithelial cells and drive tumorigenesis towards claudin-low tumors in transgenic mice. PLoS Genet 8, e1002723

29 Wellner, U., et al. (2009) The EMT-activator ZEB1 promotes tumorigenicity by repressing stemness-inhibiting microRNAs. Nat Cell Biol 11, 1487-1495

30 Yang, M.H., et al. (2010) Bmi1 is essential in Twist1-induced epithelialmesenchymal transition. Nat Cell Biol 12, 982-992

31 Hwang, W.L., et al. (2014) MicroRNA-146a directs the symmetric division of Snaildominant colorectal cancer stem cells. Nat Cell Biol 16, 268-280

32 Brabletz, S., et al. (2011) The ZEB1/miR-200 feedback loop controls Notch signalling in cancer cells. EMBO J 30, 770-782

33 Guen, V.J., et al. (2017) EMT programs promote basal mammary stem cell and tumor-initiating cell stemness by inducing primary ciliogenesis and Hedgehog signaling. Proc Natl Acad Sci U S A

34 Wu, M.J., et al. (2019) Epithelial-Mesenchymal Transition Directs Stem Cell Polarity via Regulation of Mitofusin. Cell Metab 29, 993-1002 e1006

35 Shimono, Y., et al. (2009) Downregulation of miRNA-200c links breast cancer stem cells with normal stem cells. Cell 138, 592-603

36 Valk-Lingbeek, M.E., et al. (2004) Stem cells and cancer; the polycomb connection. Cell 118, 409-418

37 Siddique, H.R. and Saleem, M. (2012) Role of BMI1, a stem cell factor, in cancer recurrence and chemoresistance: preclinical and clinical evidences. Stem Cells 30, 372-378 
38 Goetz, S.C. and Anderson, K.V. (2010) The primary cilium: a signalling centre during vertebrate development. Nat Rev Genet 11, 331-344

39 Liu, S., et al. (2006) Hedgehog signaling and Bmi-1 regulate self-renewal of normal and malignant human mammary stem cells. Cancer Res 66, 6063-6071

40 Zhou, M., et al. (2016) LncRNA-Hh Strengthen Cancer Stem Cells Generation in Twist-Positive Breast Cancer via Activation of Hedgehog Signaling Pathway. Stem Cells 34, 55-66

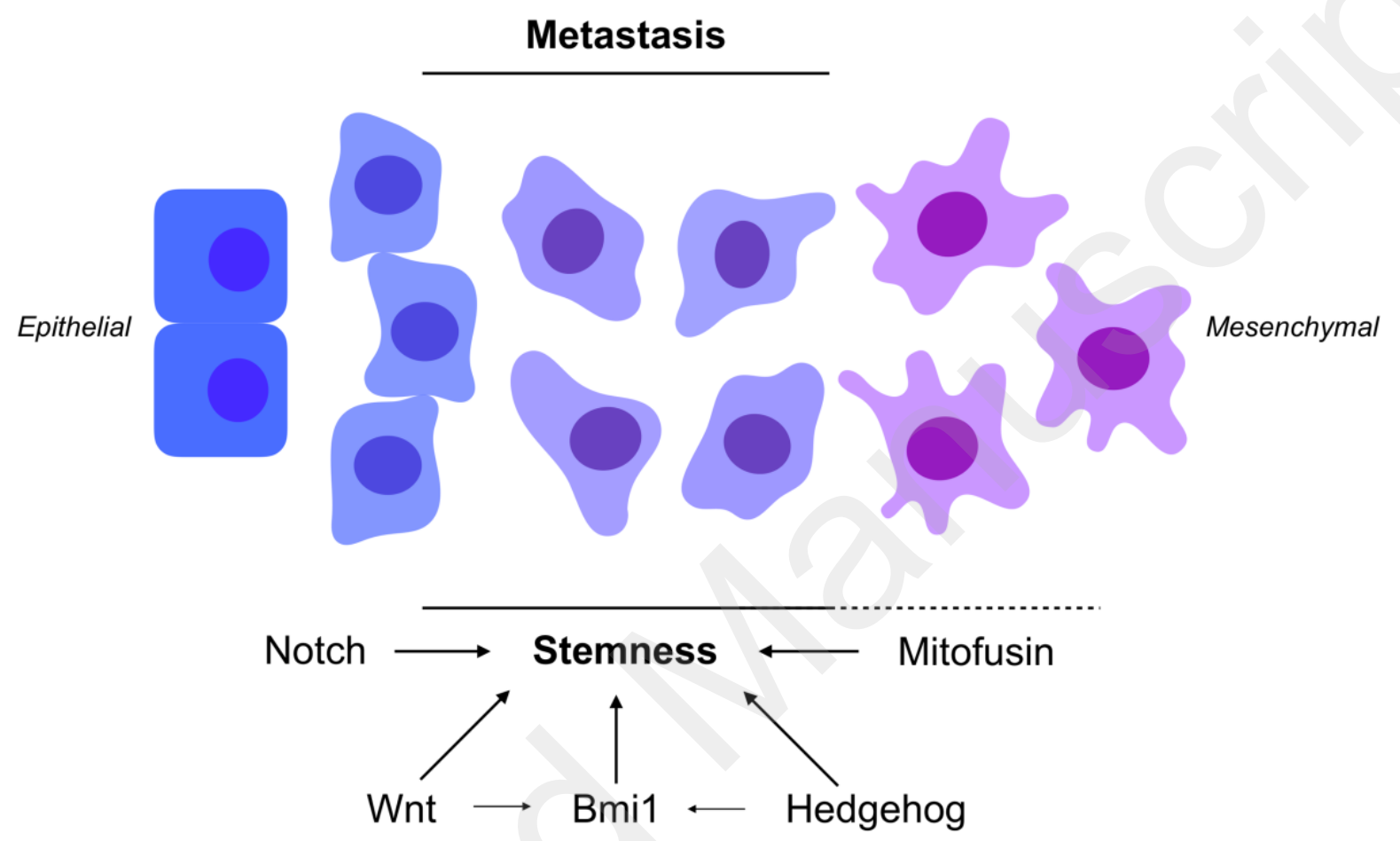

Figure 1. Key Figure. EMT programs induce stemness through different mechanisms. EMT supports multiple aspects of tumorigenesis, including tumor initiation through activation of stemness, and metastasis through induction of cell migration and invasion programs. Cells that acquire an intermediate epithelialmesenchymal identity gain the ability to metastasize to distant tissues. The precise transition states in which EMT programs induce stemness remain to be thoroughly defined. However, significant progress has been made in identifying the mechanisms by which EMT programs promote stemness including involvement of Hedgehog, Wnt, Notch, Mitofusin signaling pathways, as well as the epigenetic regulator Bmi1. 


\section{Highlights}

- Induction of EMT programs in numerous tissues is known to correlate with activation of SC properties in both normal and transformed cells.

- While many studies have defined the association between EMT induction and acquisition of stemness, few have addressed the mechanisms by which EMT directly induces SC programs.

- Recent studies have concluded that EMT can directly promote stemness through signaling pathways that activate well-established SC regulators, which control SC epigenetic reprogramming and asymmetric cell division.

- Further characterization of the precise mechanisms through which EMT promotes SC properties could reveal novel molecular vulnerabilities for cancer therapeutics.

\section{Outstanding Questions}

- Which precise EMT transition stages are associated with stemness?

- To what extent are the established mechanisms of EMT-induced stemness generalizable to other tissues and tumor types?

- Are signaling pathways that mediate EMT-induced stemness targetable for cancer therapeutics?

- Is there a therapeutic window for targeting EMT in cancer without impacting normal tissue physiology? 


\section{Glossary}

Epithelial-mesenchymal transition (EMT): the reversible, multi-step loss of epithelial traits and gain of mesenchymal traits.

Mammosphere: 3D sphere of undifferentiated cells formed by mammary epithelial cells.

Self-renewal: the ability of stem cells to divide and regenerate themselves to maintain a stem cell pool in normal or transformed tissues.

Stemness: the ability of either a normal or transformed cell to self-renew and produce differentiated progeny.

Tumorsphere: 3D sphere of undifferentiated cells formed by tumor cells. 